\title{
VACTERL/caudal regression/Currarino syndrome-like malformations in mice with mutation in the proprotein convertase Pcsk5
}

\author{
Dorota Szumska, ${ }^{1,10}$ Guido Pieles, ${ }^{1,10}$ Rachid Essalmani, ${ }^{2}$ Michal Bilski, ${ }^{1}$ Daniel Mesnard, ${ }^{3}$ \\ Kulvinder Kaur, ${ }^{4}$ Angela Franklyn, ${ }^{1}$ Kamel El Omari, ${ }^{4}$ Joanna Jefferis, ${ }_{1}^{1}$ Jamie Bentham, ${ }_{1}$ \\ Jennifer M. Taylor, ${ }^{4}$ Jurgen E. Schneider, ${ }^{1}$ Sebastian J. Arnold, ${ }^{4}$ Paul Johnson, ${ }^{5}$ \\ Zuzanna Tymowska-Lalanne, ${ }^{6}$ Dave Stammers, ${ }^{4}$ Kieran Clarke, ${ }^{7}$ Stefan Neubauer, ${ }^{1}$ Andrew Morris, ${ }^{4}$ \\ Steve D. Brown, ${ }^{6}$ Charles Shaw-Smith, ${ }^{8}$ Armando Cama, ${ }^{9}$ Valeria Capra, ${ }^{9}$ Jiannis Ragoussis, ${ }^{4}$ \\ Daniel Constam, ${ }^{3}$ Nabil G. Seidah, ${ }^{2}$ Annik Prat, ${ }^{2}$ and Shoumo Bhattacharya ${ }^{1,11}$ \\ ${ }^{1}$ Department of Cardiovascular Medicine and Wellcome Trust Centre for Human Genetics, University of Oxford, \\ Oxford OX3 7BN, United Kingdom; ${ }^{2}$ Laboratory of Biochemical Neuroendocrinology, Clinical Research Institute of \\ Montreal, Montreal, Quebec H2W 1R7, Canada; ${ }^{3}$ Swiss Institute for Experimental Cancer Research-École Polytechnique \\ Fédérale de Lausanne (ISREC-EPFL), Swiss Federal Institute of Technology, CH-1066 Epalinges, Lausanne, Switzerland; \\ ${ }^{4}$ Wellcome Trust Centre for Human Genetics, Oxford OX3 7BN, United Kingdom; ${ }^{5}$ Nuffield Department of Surgery, \\ University of Oxford, Oxford OX3 9DU, United Kingdom; ${ }^{6}$ Medical Research Council (MRC) Mammalian Genetics Unit, \\ Harwell OX11 0RD, United Kingdom; ${ }^{7}$ Department of Physiology, Anatomy, and Genetics, University of Oxford, \\ Oxford OX1 3QX, United Kingdom; ${ }^{8}$ Department of Medical Genetics, Addenbrooke's Hospital, Cambridge CB2 0XY, \\ United Kingdom; ${ }^{9}$ Unitá Operativa Neurochirurgia, Istituto G. Gaslini, 16148 Genova, Italy
}

We have identified an ethylnitrosourea (ENU)-induced recessive mouse mutation (Vcc) with a pleiotropic phenotype that includes cardiac, tracheoesophageal, anorectal, anteroposterior patterning defects, exomphalos, hindlimb hypoplasia, a presacral mass, renal and palatal agenesis, and pulmonary hypoplasia. It results from a C470R mutation in the proprotein convertase PCSK5 (PC5/6). Compound mutants (Pcsk5 $\left.{ }^{\text {Vcc/null }}\right)$ completely recapitulate the Pcsk5 $5^{V c / V c c}$ phenotype, as does an epiblast-specific conditional deletion of Pcsk5. The C470R mutation ablates a disulfide bond in the $P$ domain, and blocks export from the endoplasmic reticulum and proprotein convertase activity. We show that GDF11 is cleaved and activated by PCSK5A, but not by PCSK5A-C470R, and that Gdf11-deficient embryos, in addition to having anteroposterior patterning defects and renal and palatal agenesis, also have a presacral mass, anorectal malformation, and exomphalos. Pcsk5 mutation results in abnormal expression of several paralogous Hox genes (Hoxa, Hoxc, and Hoxd), and of Mnx1 (HIxb9). These include known Gdf11 targets, and are necessary for caudal embryo development. We identified nonsynonymous mutations in PCSK5 in patients with VACTERL (vertebral, anorectal, cardiac, tracheoesophageal, renal, limb malformation OMIM 192350) and caudal regression syndrome, the phenotypic features of which resemble the mouse mutation. We propose that Pcsk5, at least in part via GDF11, coordinately regulates caudal Hox paralogs, to control anteroposterior patterning, nephrogenesis, skeletal, and anorectal development.

[Keywords: Mouse; proprotein convertase; Gdf11; Hox; VACTERL]

Supplemental material is available at http://www.genesdev.org.

Received March 12, 2008; revised version accepted April 1, 2008.

Birth defects are a leading cause of mortality and morbidity, accounting for one in five of all infant deaths in the USA (Petrini et al. 2002), but the genetic basis for most sporadic congenital malformations are poorly un-

\footnotetext{
${ }^{10}$ These authors contributed equally to this work.

${ }^{11}$ Corresponding author.

E-MAIL shoumo.bhattacharya@well.ox.ac.uk; FAX 44-1865-287742.

Article is online at http://www.genesdev.org/cgi/doi/10.1101/gad.479408.
}

derstood. The anatomical similarities and phylogenetic proximity between the mouse and human make the mouse an ideal model for understanding the genetic and developmental basis of human congenital malformations. Both genotype-driven (knockout) and phenotypedriven mutagenesis approaches are commonly used to create novel mouse models. Unlike genotype-driven approaches, phenotype-driven screens begin with random 
mutagenesis, and make no a priori assumptions about the nature of the genes involved in development. The point mutagen ethylnitrosourea (ENU) has been successfully used in screens for mouse developmental malformations (Herron et al. 2002; Yu et al. 2004; Bogani et al. 2005; Garcia-Garcia et al. 2005), and has certain advantages over genotype-driven knockout approaches. ENU results in loss-of-function, partial loss-of-function (i.e., hypomorphic) and gain-of-function alleles that are similar to the spectrum observed in the naturally occurring mutants causing human disease. These alleles can also allow in vivo investigation of the functional consequences of abnormal protein structure (for review, see Papathanasiou and Goodnow 2005).

Given an appropriate screening method, a phenotypedriven screen could be used to identify the genetic mechanisms underlying any developmental malformation. We established a recessive three-generation ENU mutagenesis screen that uses high-throughput magnetic resonance imaging (MRI) of mouse embryos (Schneider et al. 2004) at $15.5 \mathrm{~d}$ post-coitum (dpc) as the primary screening technique to identify cardiac developmental malformations. In this screen we identified a recessive mutation $(V c c)$ with cardiac, tracheoesophageal, anorectal, axial skeletal anteroposterior patterning defects, limb malformations, presacral mass, renal and palatal agenesis, and pulmonary hypoplasia. This pleiotropic phenotype resembles those observed in certain human syndromes: VACTERL (vertebral, anorectal, cardiac, tracheoesophageal, renal, limb malformation OMIM 192350), caudal regression syndrome (CRS, OMIM 600,145 ), and Currarino Syndrome (CS, OMIM 176450).

\section{Results}

Vcc: a novel recessive ENU mouse mutant with features of VACTERL/CRS/CS

In 11 of 57 embryos that were progeny of a G1 founder male (RECC/19), and in six of 19 embryos from tested G2 males we identified consistent externally visible features including short hindlimbs, absent tail, and exomphalos (Fig. 1). In 15 embryos analyzed by MRI we observed palatal agenesis, abnormal tracheoesophageal septation, cardiac malformation, and small lungs (Figs. 1, 2). Cardiac malformations included dextroposition (eight of 15), atrial septal defects (six of 15), ventricular septal defects (15 of 15), double-outlet right ventricle (10 of 15), common arterial trunk (five of 15), hypoplastic arterial duct (two of 15), aortic vascular ring (three of 15), and rightsided aortic arch (two of 15). Measurement of lung volumes showed that the left lung was significantly hypoplastic (Fig. 2). All embryos had a complete absence of kidneys (although adrenal glands were present), and had a mass protruding ventrally from the caudal end of the spinal cord and vertebral column (Fig. 3). This mass distorted normal anorectal and bladder anatomy, and threedimensional (3D) reconstruction showed that the anus and rectum could not be identified (Fig. 3). Examination of skeletal morphology (two mutant embryos studied)
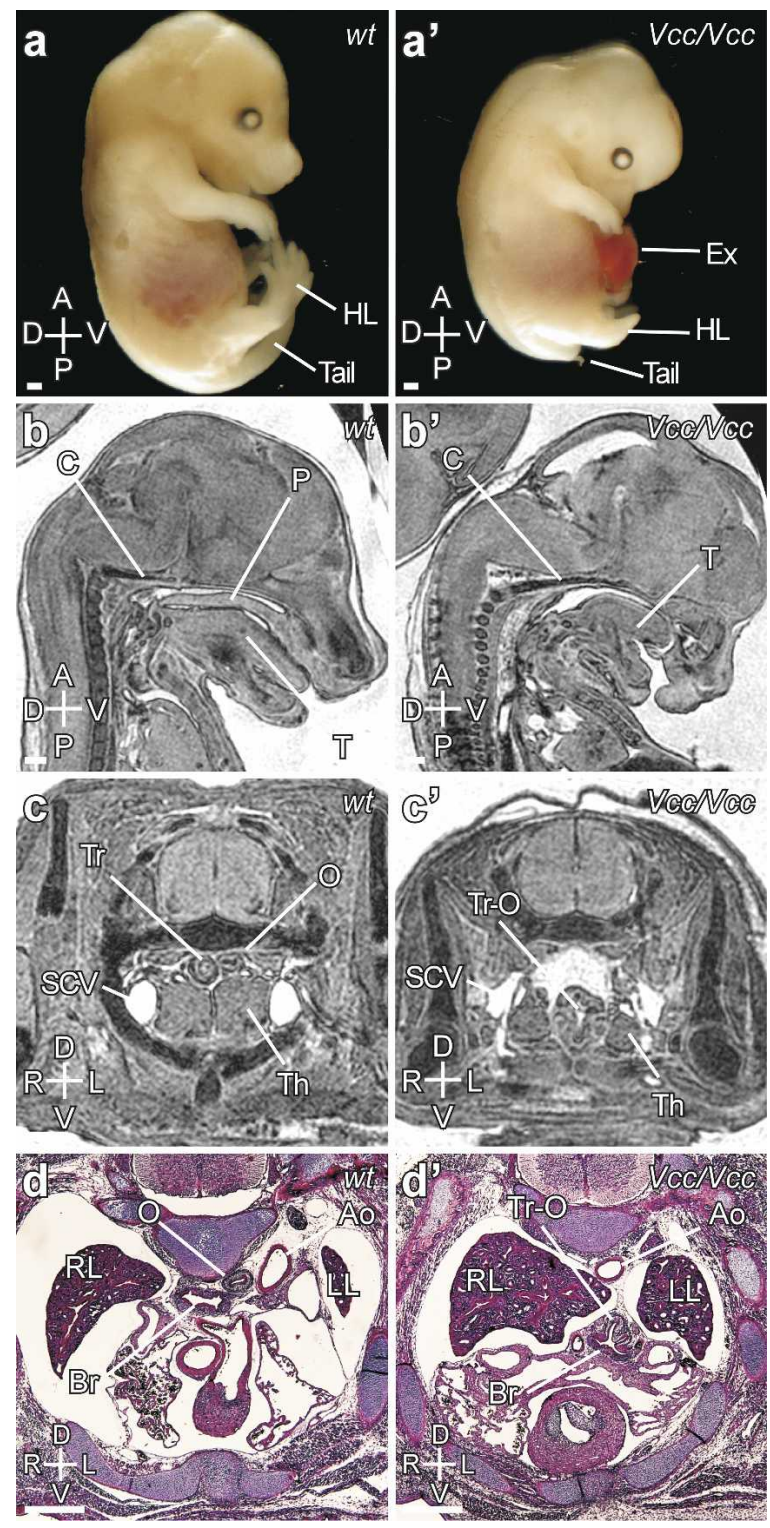

Figure 1. External appearance, palatal, and tracheoesophageal malformations at $15.5 \mathrm{dpc} .\left(a, a^{\prime}\right)$ External appearances of control littermate and mutant $(V c c / V c c)$ embryos, respectively. The mutant embryo is smaller, has a prominent exomphalos (Ex) and markedly shortened hindlimbs (HL), and lacks a tail. (b) MRI sagittal section through a control embryo showing a normal palate $(\mathrm{P})$. The tongue $(\mathrm{T})$ and clivus $(\mathrm{C})$ are indicated. $\left(b^{\prime}\right)$ Corresponding section through a littermate mutant $V c c / V c c$ embryo showing complete absence of the palate. (c) MRI transverse section through a control embryo showing the normal thymus (Th), esophagus $(\mathrm{O})$, trachea $(\mathrm{Tr})$, and superior caval vein (SCV). $\left(c^{\prime}\right)$ Corresponding section through a littermate mutant $V c c / V c c$ embryo showing that the two thymus lobes are smaller and separated, and that the trachea and esophagus are a single unseparated structure (Tr-O). (d) Transverse histological section through a wild-type embryo showing the bronchi $(\mathrm{Br})$ originating from the trachea. The esophagus $(\mathrm{O})$ and aorta $(\mathrm{Ao})$ are indicated. $\left(d^{\prime}\right)$ Corresponding section through a littermate mutant $V c c / V c c$ embryo showing the bronchi $(\mathrm{Br})$ originating from the unseparated tracheoesophagus (Tr-O). Axes are anterior (A), posterior $(\mathrm{P})$, right $(\mathrm{R})$, left $(\mathrm{L})$, dorsal $(\mathrm{D})$, and ventral $(\mathrm{V})$. Bar, $0.5 \mathrm{~mm}$. 
showed absence of caudal and sacral vertebrae, normal lumbar and cervical vertebral numbers, and 18 instead of 13 thoracic vertebrae (Fig. 4), giving a total of 31 instead of the normal 26 presacral vertebrae. Mutant embryos also had increased numbers of true ribs, and lacked a xiphisternum. The pelvic bones, femur, and tibia were severely hypoplastic. The patella, fibula, and bones of the foot were not visible. The forelimbs were mildly hypoplastic.

Vcc results from a mutation in Pcsk 5 with potential structural consequences

We mapped a minimal homozygous segregating interval to between D19MIT128 and rs13483551 (Chr 19: 17317664-18953883). Two genes in this interval (Gcnt1 and Trpm6) could be excluded as null mutations are viable (Ellies et al. 1998; Walder et al. 2002). We sequenced
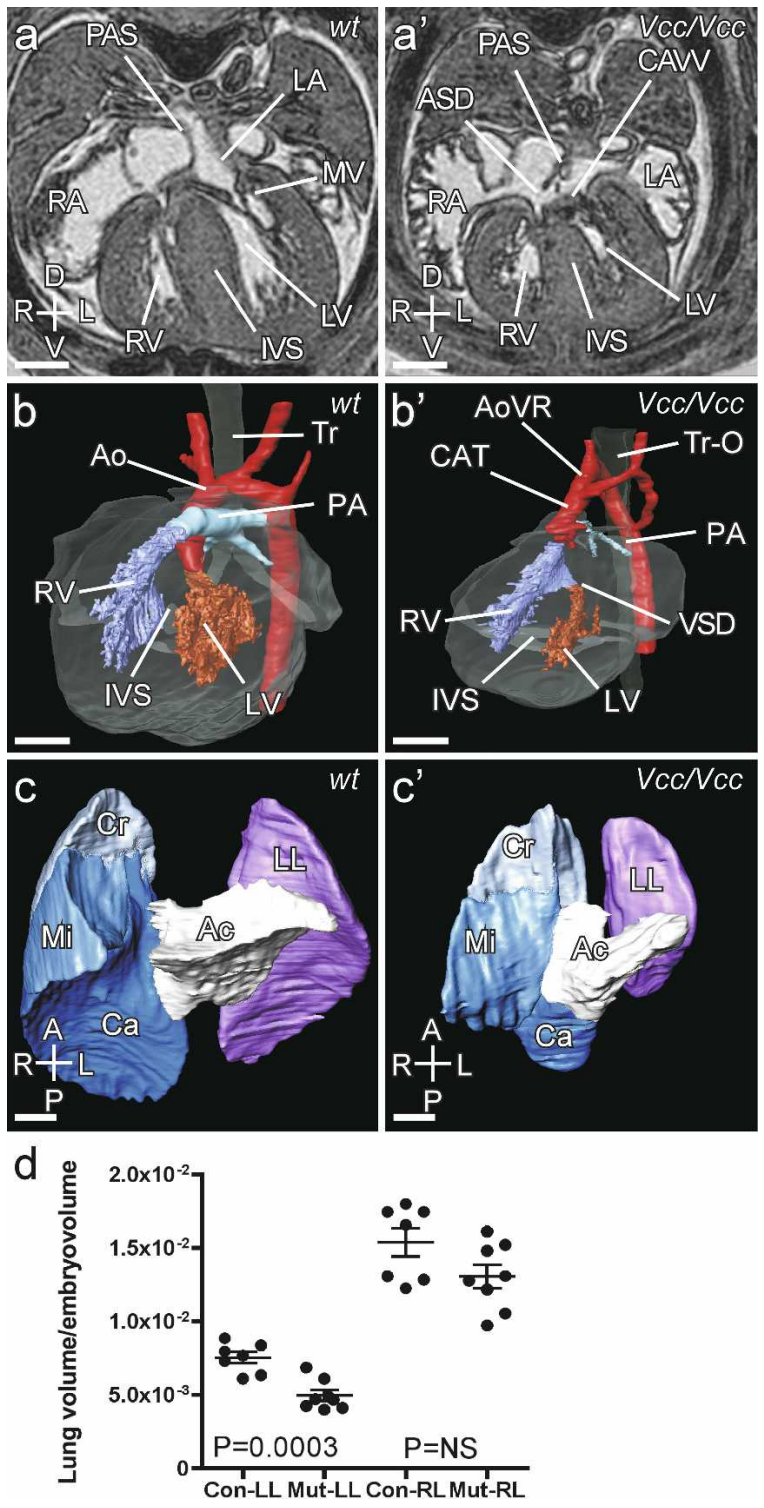

the exons of all other genes in this interval, and identified a homozygous T/C transition in Pcsk5 exon 11 (Supplemental Fig. 1). The mutation predicted a C470R amino acid change. PCSK5 is a proprotein convertase, a class of enzymes that cleave proproteins and prohormones at the consensus sequence (K/R)-(Xn)-(K/R) $\downarrow$ (for review, see Seidah and Chretien 1999). PCSK5 has two splice isoforms, A and B (Supplemental Fig. 1). PCSK5A (exons 1-21a) is soluble and is sorted to regulated secretory granules, whereas PCSK5B (exons 1-20, 21b, and 22-38) is membrane-bound, and located in the Golgi apparatus (De Bie et al. 1996; Xiang et al. 2000). The C470R mutation would be predicted to affect both splice isoforms. By analogy with FURIN, PCSK5-C470 is in the P domain, and is predicted to form a disulfide bond with C496 (Henrich et al. 2005). Molecular modeling of FURIN reveals that an analogous mutation (C450R) would result in displacement of the loop (471-482) away from P $\beta 1$ a (Supplemental Fig. 1).

\section{Vcc does not complement a Pcsk5 deletion allele}

To determine if the $V c c$ mutation complements the loss of Pcsk5, we crossed $V c c$ females to males bearing a targeted deletion of Pcsk5 exon $1\left(P c s k 5^{\Delta 1}\right)$ (Essalmani et al. 2008) and examined the phenotype of double and single heterozygotes by magnetic resonance imaging. We found that double heterozygotes $\left(P c s k 5^{V c c / \Delta 1}\right)$ completely recapitulated the $V c c / V c c$ phenotype in all embryos studied (eight of eight) (Supplemental Fig. 2). All double heterozygote embryos had hypoplastic hindlimbs, absent tail, exomphalos, presacral mass, palatal agenesis, cardiac

Figure 2. Cardiac and pulmonary malformations at $15.5 \mathrm{dpc}$. (a) MRI transverse section through a control embryo (at 15.5 dpc) showing a normal heart. The right and left atria and ventricles (RA, LA, RV, LV), mitral valve (MV), primary atrial septum (PAS), and interventricular septum (IVS) are indicated. $\left(a^{\prime}\right)$ Corresponding section of a mutant littermate $V c c / V c c$ embryo showing an ostium primum atrial septal defect (ASD) and common atrioventricular valve (CAVV). (b) Three-dimensional reconstruction of a control heart, left anterior oblique view. The aorta (Ao) arises from the left ventricle (LV), and the main pulmonary artery $(\mathrm{PA})$ rises from the right ventricle $(\mathrm{RV})$. The aortic arch passes to the left of the trachea, over the left main bronchus. $\left(b^{\prime}\right)$ Corresponding reconstruction of a mutant littermate $V c c / V c c$ heart showing a ventricular septal defect (VSD); a common arterial trunk (CAT) arising from the right ventricle $(\mathrm{RV})$, giving rise to both cranial and pulmonary arteries; and an aortic vascular ring (AoVR). The trachea is not separated from the esophagus (Tr-O). $\left(c, c^{\prime}\right)$ Three-dimensional reconstruction of lungs from wild-type $(\mathrm{wt})$ and littermate mutant $(V c c / V c c)$ embryos. The cranial $(\mathrm{Cr})$, middle $(\mathrm{mi})$, caudal $(\mathrm{Ca})$, and accessory (Ac) lobes of the right lung and the left lung (LL) are indicated. (d) Lung volumes (mean \pm SEM, corrected for embryo volume) are shown for control left and right lungs (Con-LL, Con$\mathrm{RL}$ ), and littermate mutant $V c c / V c c$ left and right lungs (MutLL, Mut-RL). The mutant left lung is significantly smaller than the control $(t$-test, $P=0.003)$. Axes are anterior $(\mathrm{A})$, posterior $(\mathrm{P})$, right $(\mathrm{R})$, left $(\mathrm{L})$, dorsal $(\mathrm{D})$, and ventral $(\mathrm{V})$. Bars, $0.5 \mathrm{~mm}$. 
and tracheoesophageal malformations, pulmonary hypoplasia, and renal agenesis. The cardiac malformations included dextroposition (two of eight), ventricular septal defect (eight of eight), double-outlet right ventricle (six of eight), atrial septal defect (three of eight), common arterial trunk (two of eight), and aortic vascular ring (one of eight). No abnormalities were observed in single heterozygous control embryos. These results show that that Vcc does not complement Pcsk5 $5^{\Delta 1}$

\section{Epiblast-specific deletion of Pcsk5 recapitulates the Vcc phenotype}

Pcsk5 is expressed in maternal-derived, hypoblast-derived, and epiblast-derived tissues (Constam et al. 1996; Rancourt and Rancourt 1997). To determine the role of epiblastic Pcsk5 in organogenesis and patterning we made use of a Pcsk $5^{\text {flox }}$ allele that deletes exon 1 by Cre-mediated recombination (Essalmani et al. 2008). We used this allele to delete Pcsk5 specifically in the epiblast using Meox2Cre and studied the phenotype of embryos (Meox2Cre; Pcsk5 $5^{\Delta 1 / \text { flox })}$ at $15.5 \mathrm{dpc}$ using MRI (Supplemental Fig. 3). We found that this recapitulated the $V c c / V c c$ phenotype, but with reduced penetrance, likely reflecting incomplete deletion of Pcsk5. Meox2Cre; Pcsk $5^{\Delta 1 / f l o x}$ embryos had hypoplastic hindlimbs (four of four), absent or hypoplastic tail (four of four), cardiac malformations (four of four), palatal agenesis (three of four), tracheoesophageal malformation (two of four), pulmonary hypoplasia affecting left lung (two of four), exomphalos (three of four), and renal agenesis (three of four). The cardiac malformations included dextroposition (one of four), transposition of great arteries (one of four), double-outlet right ventricle (three of four),

Figure 3. Visceral malformations. (a) MRI transverse sections through the abdomen of a wild-type embryo (wt) at $15.5 \mathrm{dpc}$, showing the normal kidneys (LK, left kidney), ureter (LU, left ureter) and adrenals (LAd, left adrenal). ( $\left.a^{\prime}\right)$ Corresponding sections through a mutant littermate $V c c / V c c$ embryo. The kidneys and ureter could not be identified. The pancreas $(\mathrm{P})$ is indicated. (b) MRI sagittal sections through a 15.5 -dpc control embryo showing the normal urinary bladder (UB), urethra (U), liver (Li), and vertebral column (VC). The tail (T) is indicated. $\left(b^{\prime}\right)$ Corresponding section through a mutant littermate $V c c /$ $V c c$ embryo. A presacral mass (PM) is seen between the vertebral column and the bladder. The liver herniates through the umbilicus forming an exomphalos (Ex). (c) Three-dimensional reconstruction of a control embryo showing the spinal cord $(\mathrm{SC})$, gut $(\mathrm{G})$, urinary bladder $(\mathrm{UB})$, urethra $(\mathrm{U})$, and rectum $(\mathrm{R})$. $\left(c^{\prime}\right)$ Corresponding reconstruction of a mutant $V c c / V c c$ embryo showing that the presacral mass (PM) is connected to the spinal cord, and its relation to the bladder and gut. The rectum could not be defined. (d) Sagittal histological section of a 13.5-dpc wild-type embryo showing the vertebral column (VC) and the spinal cord (SC) entering the tail $(\mathrm{T}) .\left(d^{\prime}\right)$ Corresponding section of a littermate mutant $V c c / V c c$ embryo showing the vertebral column and spinal cord entering the presacral mass (PM). Axes are anterior $(\mathrm{A})$, posterior $(\mathrm{P})$, right $(\mathrm{R})$, left $(\mathrm{L})$, dorsal $(\mathrm{D})$, and ventral (V). Bars, $0.5 \mathrm{~mm}$. ventricular septal defect (four of four), atrial septal defect (two of four), right-sided aortic arches (two of four), and aortic vascular ring (one of four). A presacral mass distorting anorectal and bladder anatomy was observed in the two embryos that lacked a visible external tail. Meox2Cre; Pcsk5 $5^{\Delta 1 / f l o x}$ embryos also had axial and appendicular skeletal defects similar to $V c c / V c c$ (Essalmani et al. 2008).

\section{PCSK5A C470R mutation affects its secretion and localization}

To investigate the functional consequences of the C470R mutation we transfected PCSK5A-Flag and PCSK5A-C470R-Flag-expressing plasmids into COS-1

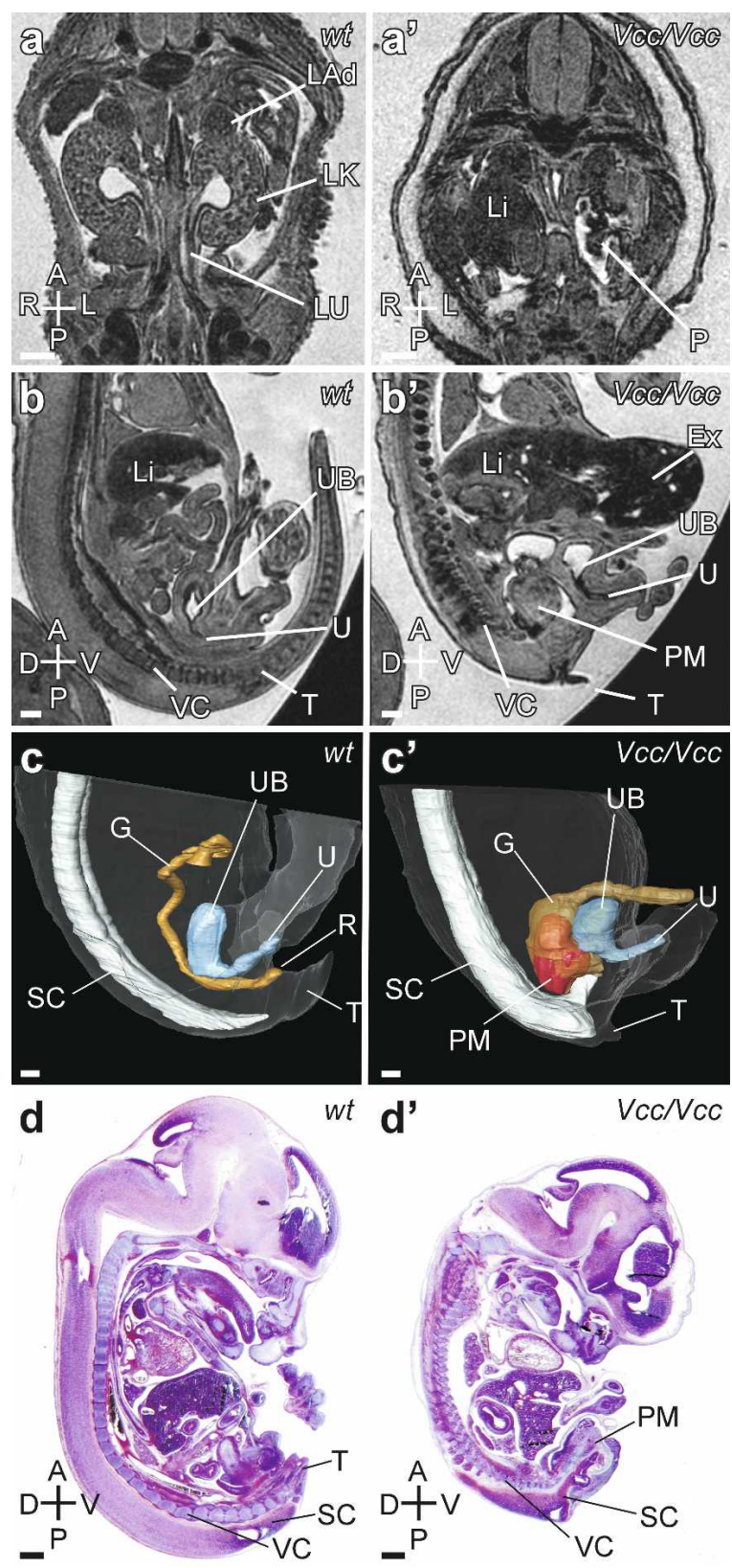



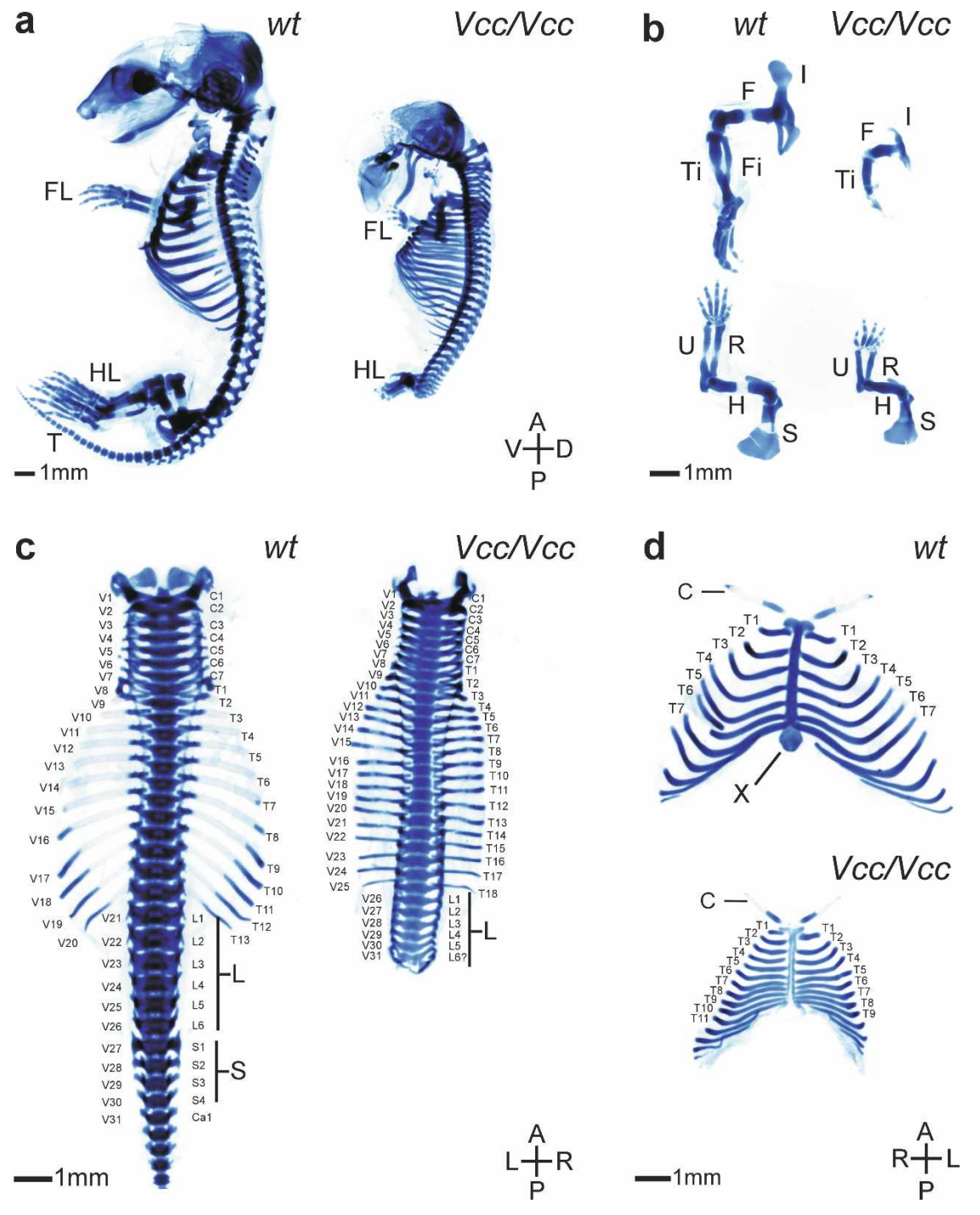

Figure 4. Skeletal malformations at 15.5 dpc. (a) Wild-type (wt) embryo showing normal tail $(\mathrm{T})$, hindlimbs (HL), and forelimbs (FL). Mutant littermate embryo $(V c c / V c c)$ showing absence of tail, and severely hypoplastic hindlimbs. The forelimbs are relatively spared. $(b, t o p)$ Left hindlimb from the wild-type embryo (left), and the mutant embryo (right). (Bottom) The ilium (I), femur (F), tibia (Ti), and fibula (Fi) are indicated. Wild-type (left) and mutant (right) forelimb. Ulna (U), radius $(\mathrm{R})$, humerus $(\mathrm{H})$, and scapula $(\mathrm{S})$ are indicated. $(c)$ Axial skeletons from wildtype and littermate mutant $V c c / V c c$ embryos, respectively. Vertebrae are numbered by craniocaudal position (left), and by identity (right). The wild-type embryo has seven cervical (C1-C7), 13 thoracic (T1-T13, identified by the presence of ribs), six lumbar (L1-L6), and four sacral (S1-S4) vertebrae. The first caudal vertebra (Ca1) is also indicated. There are 26 presacral vertebrae. The mutant embryo has 18 thoracic vertebrae, and lacks sacral and caudal vertebrae, and has a total of 31 presacral vertebrae. (d) Rib cage from the wild-type embryo (top), and the mutant embryo (bottom). In the wild-type embryo, the clavicle $(\mathrm{C})$ and xiphisternum $(\mathrm{X})$ are indicated, as are the true ribs (T1-T7, joining the sternum directly). The mutant embryo lacks the xiphisternum, has 11 true ribs on the right, and nine true ribs on the left. Axes are anterior $(\mathrm{A})$, posterior $(\mathrm{P})$, right $(\mathrm{R})$, eft $(\mathrm{L})$, dorsal $(\mathrm{D})$, and ventral (V). Bars, $0.5 \mathrm{~mm}$.

cells, and examined the secretion and immunolocalization of the Flag-tagged peptides (Fig. 5). Immunoblotting and pulse-label radioimmunoprecipitation experiments indicated that both Flag-tagged and untagged versions of PCSK5A (wild type) are efficiently synthesized and secreted into the medium. Although tagged and untagged versions PCSK5A-C470R were also efficiently synthesized, they could not be detected in the conditioned medium. Immunofluorescence experiments showed that whereas PCSK5A-Flag is localized to the trans-Golgi network, PCSK5A-C470R-Flag localizes instead to the endoplasmic reticulum (Fig. 5).

\section{PCSK5A C470R mutation affects its enzymatic activity}

We next used cotransfection assays to examine the effect of the mutation on the cleavage of two PCSK5 substrates, BMP4 (Cui et al. 1998), and LEFTY1 (Ulloa et al. 2001). These experiments showed that whereas PCSK5A efficiently cleaves BMP4 and LEFTY1 propeptides to their mature forms, the mutant PCSK5A-C470R does not (Fig. 5). We also monitored the enzymatic activity of the conditioned medium from transfected cells using a fluorogenic peptide assay. This showed that while conditioned medium from cells transfected with wild-type PCSK5A plasmid had in vitro enzymatic activity, the activity of conditioned medium from cells transfected with PCSK5A-C470R plasmid was identical to that of vector transfected cells (Supplemental Fig. 4).

\section{Gdf11 deletion partially phenocopies Vcc}

Certain features of the $V c c$ mutant (palatal agenesis, renal agenesis, increased numbers of thoracic vertebrae to 18 , increased numbers of true ribs, absent tail) phenocopy the loss of Gdf11 (McPherron et al. 1999; Esquela and Lee 2003). We examined Gdff1 $1^{-/-}$embryos at 15.5 dpc using MRI, and confirmed that they had absent tail (five of five), palatal agenesis (five of five), and renal agen- 

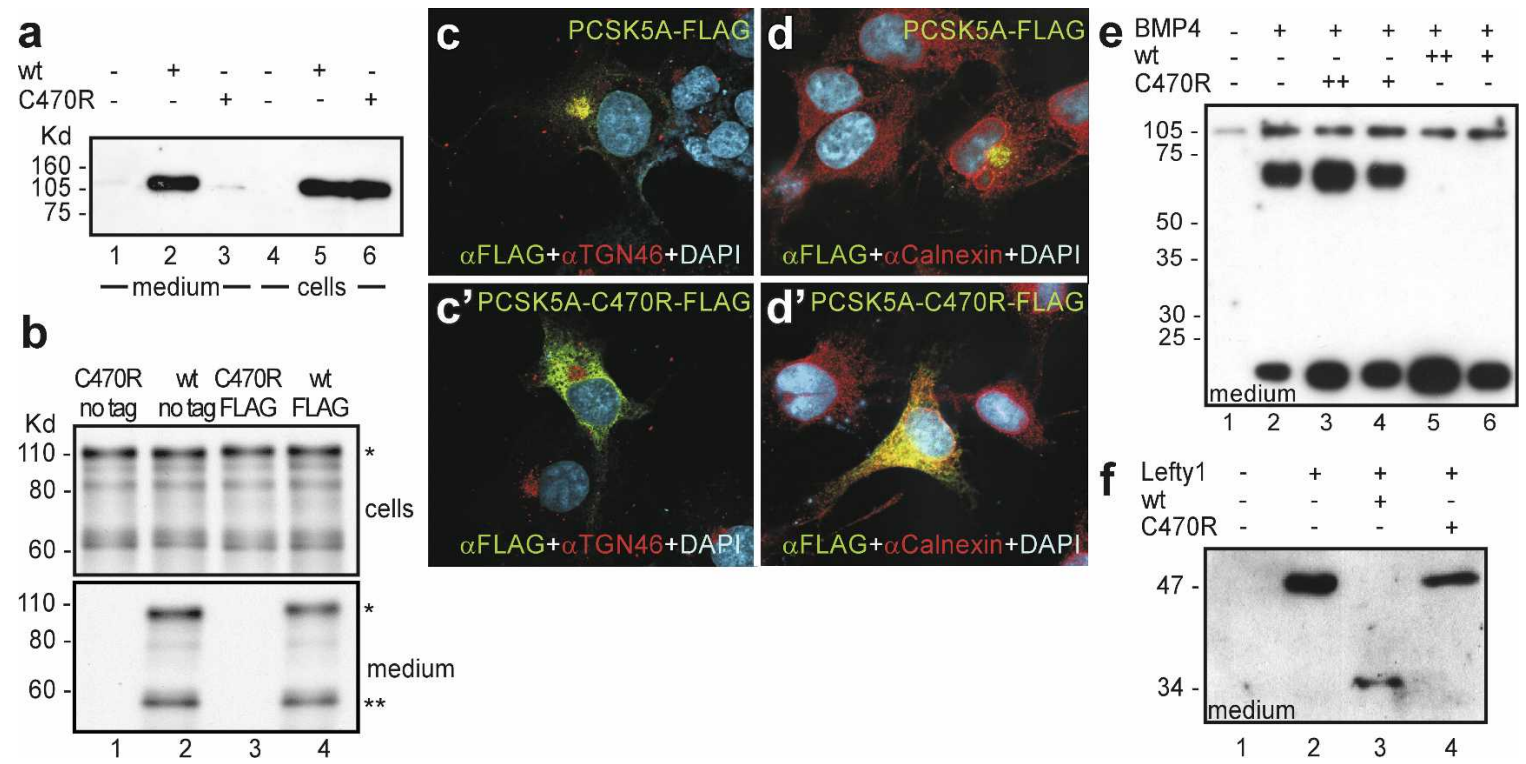

Figure 5. Functional analysis of PCSK5A and PCSK5A-C470R. (a) Western blot of Cos-1 cell extracts and conditioned medium following transfection with C-terminally Flag-tagged PCSK5A (915-amino-acid isoform, wild type) and the corresponding C470R mutant. The blot was probed with anti-Flag monoclonal antibody. ( $b$, top panel) Autoradiogram of anti-PCSK5 immunoprecipitates from HEK293 cells transfected with the indicated plasmids and radiolabeled with $\left[{ }^{35} \mathrm{~S}\right]$ Met + Cys. (Bottom panel) Autoradiogram of anti-PCSK5 immunoprecipitates from the conditioned media of the transfected cells. $\left({ }^{\star}\right)$ PCSK5A; $\left.{ }^{\star \star \star}\right)$ PCSK5A cleavage product that deletes the $\mathrm{C}$ terminus (De Bie et al. 1996). $\left(c, c^{\prime}, d, d^{\prime}\right)$ Immunofluorescence confocal microscopy of Cos-1 cells transfected with Flag-tagged PCSK5A and PCSK5A-C470R. Cells were stained with DAPI (blue) to localize nuclei, anti-Flag monoclonal antibody (green), anti-TGN46 polyclonal antibody (red, in $\left.c, c^{\prime}\right)$ to localize the trans-Golgi network, and anti-calnexin polyclonal antibody (red, in $\left.d, d^{\prime}\right)$ to localize the endoplasmic reticulum. The merged images are shown, in which colocalizing signals appear yellow. $(e)$ Western blot of Cos-1 cell conditioned medium following cotransfection with a mouse BMP2-mycBMP4 hybrid plasmid and either PCSK5A (wild type, 0.8 or $0.4 \mu \mathrm{g}$, lanes 5,6, respectively) or the corresponding C470R mutant (0.8 or $0.4 \mu g$, lanes 3,4, respectively). BMP2mycBMP4 plasmid expresses a protein containing the mouse BMP2 prodomain fused to the BMP4 mature peptide in which the BMP4 cleavage site (TRRRAKR $\downarrow S P)$ is followed by an internal myc tag. The blot was probed with anti-myc monoclonal antibody. $(f)$ Western blot of Cos-1 cell conditioned medium following cotransfection with LEFTY1 and either PCSK5A (wild type, lanes 3) or the corresponding C470R mutant (lane 4). The blot was probed with anti-LEFTY1 antibody.

esis (five of five). In addition, we found that these embryos had either an intra-abdominal (three of five) or an extra-abdominal (two of five) mass arising from the spinal cord, exomphalos (two of five), and abnormal anorectal anatomy (five of five) (Fig. 6). We found no evidence of cardiac or tracheoesophageal malformation or lung or hindlimb hypoplasia in these embryos.

\section{GDF11 is activated and cleaved by PCSK5A}

GDF11 is a TGF $\beta$ superfamily molecule (McPherron et al. 1999), and sequence analysis reveals that it contains a consensus proprotein convertase cleavage site (Duckert et al. 2004), suggesting that PCSK5 may cleave the GDF11 propeptide to its mature form. We used a cotransfection luciferase assay that detects activated GDF11 to determine if it can be activated by cotransfected PCSK5A (Yan et al. 2002; Andersson et al. 2006). In this assay we found that transfection of GDF11 alone into 293 T cells activated the reporter, likely reflecting the presence of an endogenous proprotein convertase (Fig. 6). This activity was increased threefold by cotransfecting in PCSK5A-Flag. In contrast, cotransfection of PCSK5A-
C470R-Flag did not affect reporter activity. We also assayed the ability of conditioned medium from cells transfected with PCSK5A-Flag and PCSK5A-C470R-Flag to cleave a GDF11-derived peptide containing the putative cleavage site. We found that conditioned medium from PCSK5A-Flag transfected cells achieved a percent cleavage of $58.4 \%$ (mean of two independent experiments), whereas no significant cleavage was observed with conditioned medium from PCSK5A-C470R-Flag transfected cells (Fig. 6). Taken together, these results indicate that PCSK5A can cleave and activate GDF11, and that the C470R mutation ablates this function.

\section{The Vcc mutation does not affect embryonic Pcsk5 or Gdf11 expression}

Endogenous Pcsk5 transcript could be detected in wildtype embryos, most strongly in the caudal-most somites, to a reduced extent in more anterior somites, and also strongly in the apical ectodermal ridges of the forelimb, and hindlimb buds (three of three examined) (Fig. 7), consistent with previous reports (Constam et al. 1996; Essalmani et al. 2006). This distribution was unchanged in 


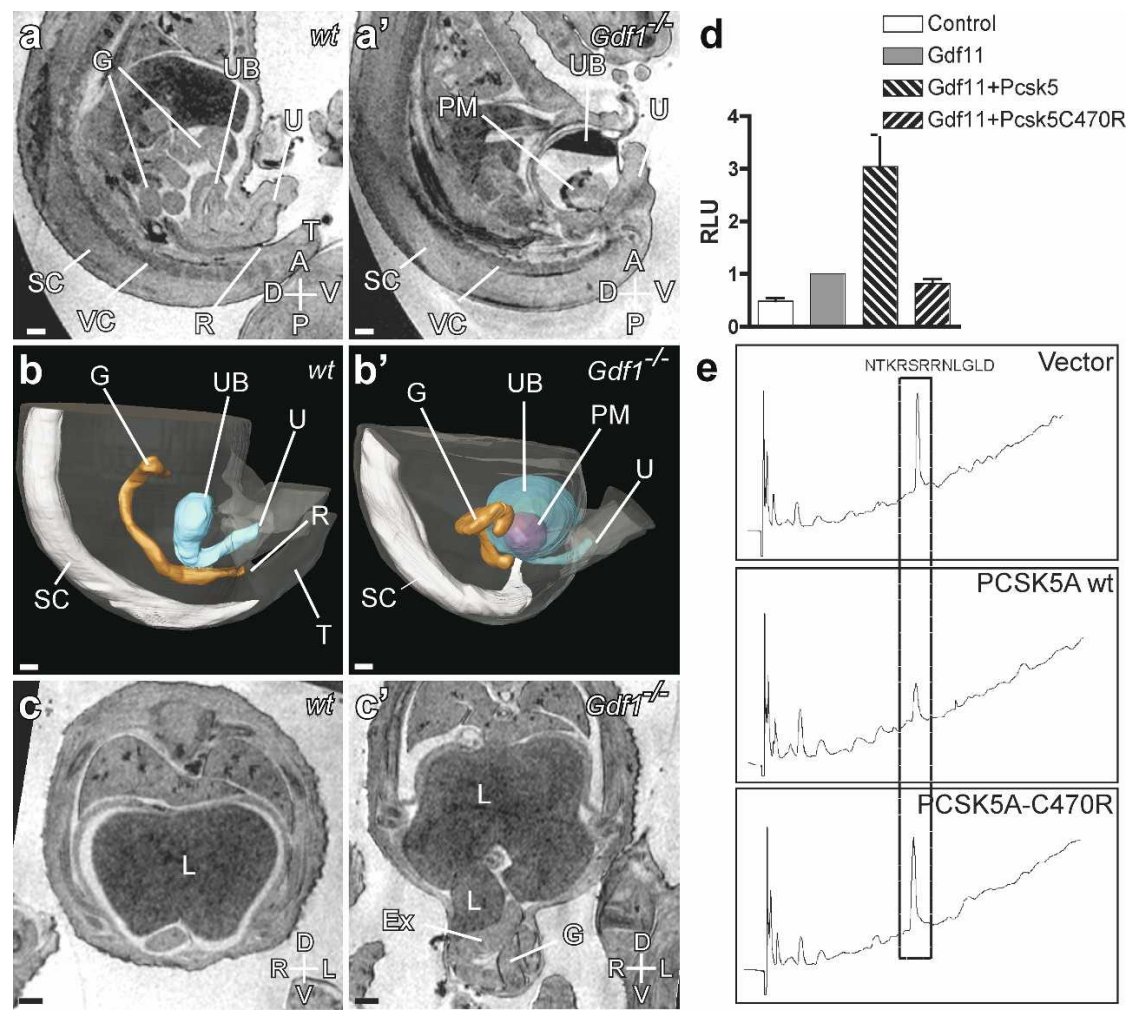

Figure 6. Phenotypic effects of $G d f 11$ deletion and its activation by PCSK5. $(A, B)$ MRI sagittal section and $3 \mathrm{D}$ reconstruction of a 15.5-dpc control wild-type (wt) embryo showing a normal urinary bladder (UB), urethra $(\mathrm{U})$, gut loops $(\mathrm{G})$, and vertebral column (VC). The tail (T) and rectum (R) are indicated. $\left(a^{\prime}, b^{\prime}\right)$ Corresponding section and $3 \mathrm{D}$ reconstruction of a mutant littermate Gdf $11^{-/-}$embryo. A presacral mass (PM) arising from the spinal cord is seen entering the bladder. The rectum could not be defined. $\left(c, c^{\prime}\right)$ MRI transverse sections of wildtype and mutant littermate $G d f 11^{-/-} \mathrm{em}$ bryo, respectively, at $15.5 \mathrm{dpc}$. The liver (L) and gut loops $(\mathrm{G})$ are present in the exom-

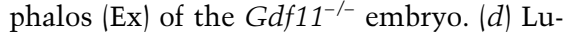
ciferase reporter assay for GDF11 activation. Flag-tagged PCSK5A-Flag wild-type and C470R mutant-expressing plasmids were cotransfected in $293 \mathrm{~T}$ cells together with plasmids expressing GDF11, and FOXH1 expression plasmids to monitor induction of the reporter construct AR3-lux. Results are presented in relative luciferase units (mean \pm SEM of three independent transfections). Values for GDF11 alone, reflecting baseline activity, were set at 1 . In this assay, PCSK5A does not activate luciferase in the absence of GDF11 (data not shown). (e) Chromatographic profiles of a 12-mer mouse GDF11-derived peptide (NTKRSRRNLGLD) containing a consensus proprotein convertase cleavage site, incubated overnight with conditioned media from vector control, PCSK5A wild-type, and PCSK5AC470R transfected HEK293 cells. Axes are anterior $(\mathrm{A})$, posterior $(\mathrm{P})$, right $(\mathrm{R})$, left $(\mathrm{L})$, dorsal $(\mathrm{D})$, and ventral $(\mathrm{V})$. Bars, $0.5 \mathrm{~mm}$.

littermate mutant $V c c / V c c$ embryos (three of three examined) (Fig. 7). We were unable to detect endogenous PCSK5 protein from mouse embryos using five commercially available antibodies by Western blotting (Supplemental Fig. 7). Endogenous Gdf11 transcript could be detected in wild-type embryos in the tailbud, and in the forelimb bud and hindlimb bud, consistent with previous observations (McPherron et al. 1999; Nakashima et al. 1999). This distribution was unchanged in littermate mutant $V c c / V c c$ embryos (three of three examined) (Fig. 7).

The Vcc mutation results in abnormal Hox and Mnx1 gene expression

Gdf11 deletion is associated with abnormal Hoxc10 and Hoxc11 expression (McPherron et al. 1999). We therefore explored the idea that Pcsk5 controls Hox expression using in situ hybridization (Fig. 7). We found that while Hoxc10 was expressed in the caudal region of wild-type embryos, it was almost absent in littermate $V c c / V c c$ mutants (three of three mutants studied) (Fig. 7). Hoxa11 was expressed in the forelimb and hindlimb buds in wild-type controls, but was markedly reduced in the littermate mutant embryos (three of three mutants studied). Hoxa10 and Hoxd11 were expressed in the tailbud region, forelimb bud, and hindlimb bud in wild-type con- trols but markedly reduced in the littermate mutant embryos (three of three mutants studied) (Fig. 7).

Using an unbiased microarray approach (Supplemental Fig. 5) we also found that the expression of several Hox genes was significantly reduced in $V c c / V c c$ mutants. These included Hoxc9, Hoxc10, Hoxd9, Hoxd10, and Hoxd12. In addition, the expression of Hoxa11os (a noncoding transcript from the opposite strand of the Hoxa locus) (Hsieh-Li et al. 1995) was also significantly reduced. Using in situ hybridization we found that the Hoxa11os was expressed in tailbud and forelimb bud in wild-type, but was markedly reduced in the littermate mutant embryos. Hoxd10 was expressed in tailbud, forelimb bud, and hindlimb bud in the wild-type, but markedly reduced in tailbud and hindlimb bud in the littermate mutant embryos (three of three mutants studied) (Fig. 7). Hoxd12 was expressed in the tailbud region, hindlimb bud, and forelimb bud in the wild-type, but undetectable in littermate mutant embryos (three of three mutants studied; Fig. 7).

In addition to Hox genes, we found that the homeobox-like gene Mnx1 (Hlxb); HB9, which is expressed in the motorneurons and specifies their identity; and the human ortholog of which is mutated in CS) (Ross et al. 1998; Arber et al. 1999; Harrison et al. 1999; Li et al. 1999; Lynch et al. 2000), was ectopically expressed in the ventral aspect of the tail bud (three of three mutants studied) (Fig. 7). In contrast, no differences were observed 
Figure 7. Gene expression analysis using in situ hybridization. Wild-type (wt) control and littermate mutant Pcsk5 ${ }^{V c c / V c c}$ $(V c c / V c c)$ embryos, respectively, are shown at the time points indicated. $\left(a, a^{\prime}\right)$ Pcsk5 is expressed in the caudal-most somites (S), and the hindlimb and forelimb buds (in the apical ectodermal ridge) in both wild-type and mutant embryos. $\left(b, b^{\prime}\right)$ Gdf11 is expressed strongly in the tailbud (TB), hindlimb bud (HLB), and forelimb bud (FLB) in both wild type and mutant. $\left(c, c^{\prime}\right)$ Hoxa10 is expressed in the otic vesicle (OV), forelimb bud (FLB), hindlimb bud (HLB), tailbud region (TB), and caudal end of the wild-type embryo, but is reduced in the mutant. $\left(d, d^{\prime}\right)$ Hoxa11 is expressed strongly in otic vesicle (OV), hindlimb bud (HLB), and forelimb bud (FLB) of the wild type, but is reduced in forelimb bud (FLB), and absent in hindlimb bud (HLB) of the mutant. $\left(e, e^{\prime}\right)$ Hoxa11os is expressed in otic vesicle (OV), forelimb bud (FLB), and tailbud (TB) of the wild-type embryo, but is reduced in the mutant. $\left(f, f^{\prime}\right)$ Hoxc10 is expressed strongly at the caudal end of the wild-type embryo, and the tailbud region (TB), but only weakly in the mutant embryo. $\left(g, g^{\prime}\right)$ Hoxd10 is expressed in otic vesicle (OV), forelimb bud (FLB), hindlimb bud (HLB), tailbud (TB), and caudal end of the wild-type embryo, but is reduced in the mutant. $\left(h, h^{\prime}\right)$ Hoxd11 is expressed in otic vesicle (OV), forelimb bud (FLB), hindlimb bud (HLB), tailbud (TB), and caudal end of the wild-type embryo, but is reduced in the mutant. $\left(i, i^{\prime}\right)$ Hoxd12 is expressed in otic vesicle (OV), forelimb bud (FLB), hindlimb bud (HLB), tailbud (TB), and caudal end of the wild-type embryo, but is reduced in the mutant. $\left(j, j^{\prime}\right)$ $M n x 1$ ( $H 1 \times b 99, H b 9)$ in wild-type embryos is expressed in the motor neurons of the spinal cord $(\mathrm{MN})$ and in the dorsal aspect of tailbud (arrowhead). In the mutant embryo, $M n x 1$ is expressed on the ventral aspect of the tailbud, extending apparently into the gut $(\mathrm{G})$ (arrowhead). $\left(k, k^{\prime}\right) T$ is expressed in the tailbud (TB) of both wildtype and mutant embryos. $\left(1,1^{\prime}\right) \mathrm{Tbx} 6$ is expressed in the tailbud (TB) and caudal end of both wild-type and mutant embryos. Bars, $0.5 \mathrm{~mm}$.
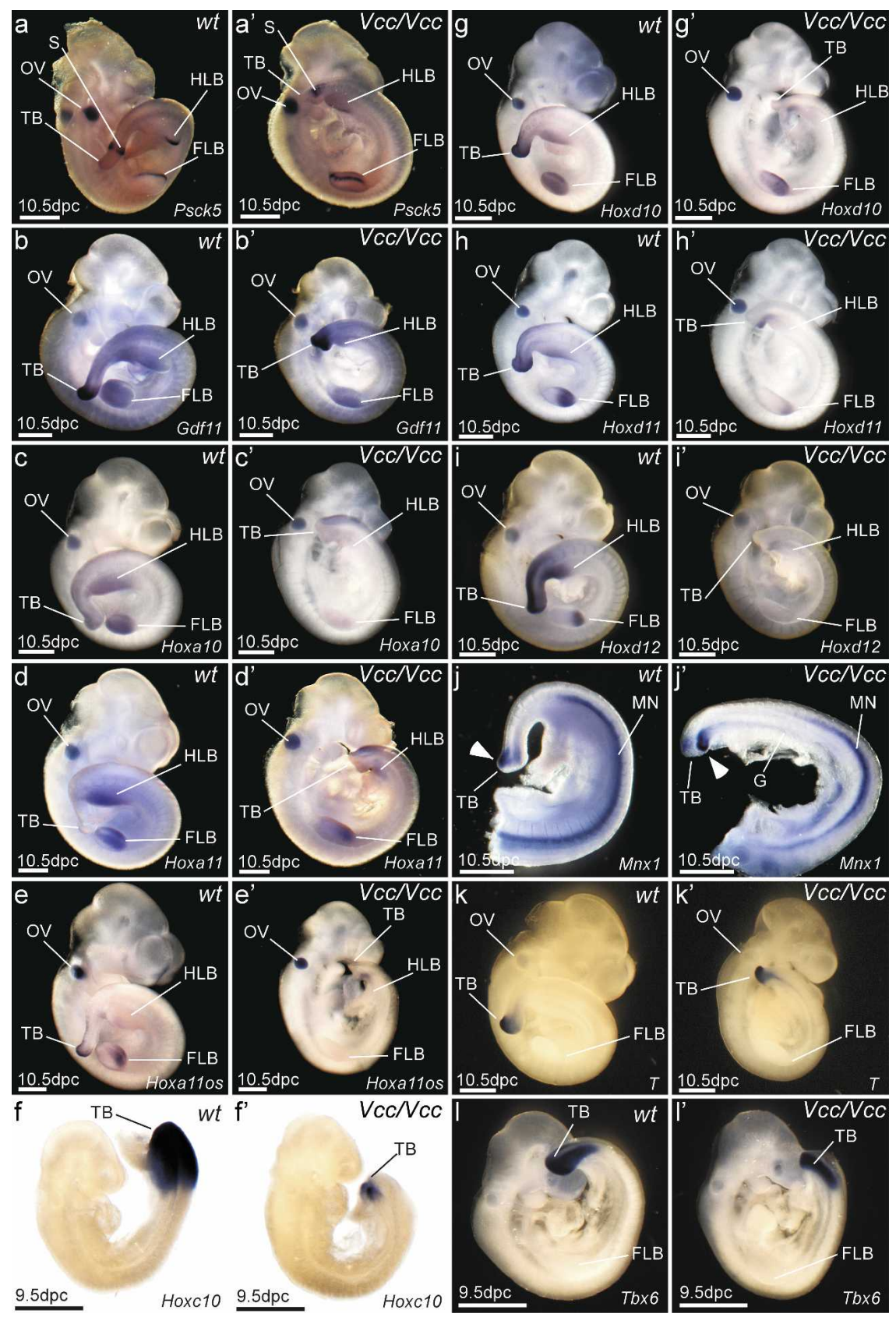

in expression of other caudal markers such as $T, T b \times 6$ (three of three mutants studied) (Fig. 7).

\section{Nonsynonymous mutations in VACTERL/CRS patients}

The phenotypic features of this mouse mutant resemble an overlap between VATER/VACTERL association, CRS, and CS. We studied European (Italian and British) patients with features of VACTERL/CRS/CS for mutations in the open reading frame of PCSK5 using amplicon DNA melting analysis (Herrmann et al. 2006) followed by sequencing. VACTERL is diagnosed when three or more features comprising the association are present in a patient (Khoury et al. 1983). In 36 patients with a VACTERL score $\geq 3$ (assigning a score of 1 for each VACTERL component) we identified four patients with nonsynonymous variants in PCSK5B (two with E1575K; 
one with E1256K, one with P1228S) (see Supplemental Table 2). E1575K was present in an Italian patient, and in a British patient. In 13 patients with CRS, but with a VACTERL score $<3$, we identified one patient with a nonsynonymous change (heterozygous V1250I).

We screened these variants in all patients and in ethnically matched controls (137 Italian and 91 CEPH-European) using an iPLEX (Sequenom) assay. Here we genotyped in addition, 59 known SNPs across PCSK5 (Supplemental Table 4). E1256K, V1250I, and P1228S were not present in the relevant exons of ethnically matched controls, indicating that they are not common polymorphisms. E1575K was present in a single Italian control. We analyzed protein sequence alignments (Supplemental Fig. 6) and found that E1256 is conserved in all tetrapod lineages where a PCSK5B sequence is available and in zebrafish. P1228 is conserved in all tetrapods apart from Xenopus. E1575 is conserved in all eutherian mammals analyzed, while V1250 is conserved in some but not all eutherian mammals. To determine the origin of the variant we screened family samples where available, and found that they were invariably inherited from a phenotypically normal parent (Supplemental Table 2). We also identified a homozygous P1228S change in a phenotypically normal sister, and a heterozygous P1228S change in a second phenotypically normal sister. We investigated haplotype association with disease using the GENEBPM software (Morris 2006). The maximum $\log _{10}$ Bayes' factor across all windows was 5.35, providing no evidence of association of haplotypes across the region with disease.

\section{Discussion}

In these studies, we identified an ENU-induced recessive mouse mutation, $V c c / V c c$, which has a pleiotropic late gestational phenotype including cardiac, tracheoesophageal, anorectal, axial skeletal, and limb malformations, exomphalos, presacral mass, renal and palatal agenesis, and pulmonary hypoplasia. It results from a C470R mutation in PCSK5 ablating a predicted disulfide bond in the P domain. We show that the Vcc allele does not complement a Pcsk5 exon1 deletion $\left(P \operatorname{csk} 5^{\Delta 1}\right)$, with double heterozygotes $\left(V c c / P c s k 5^{\Delta 1}\right)$ completely recapitulating the $V c c / V c c$ phenotype. Thus, $V c c$ is allelic to $P c s k 5^{\Delta 1}$, allowing us now to refer to it as Pcsk $5^{V c c}$. The PCSK5A-C470R mutation blocks export from the endoplasmic reticulum and proprotein convertase activity in vitro, and results in abnormal expression of several caudal Hox genes from different paralogous groups.

The observed phenotype is consistent with the known expression pattern of Pcsk5, which include the somites, vertebral cartilage primordia, dorsal surface ectoderm, the apical ectodermal ridge of limb buds, the first branchial arch, the heart, aorta, adrenals, kidney, gut, and lungs (Constam et al. 1996; Rancourt and Rancourt 1997; Essalmani et al. 2006). Importantly, in addition to its embryonic expression, Pcsk5 is also expressed in nonepiblastic tissues such as the extraembryonic endoderm, trophoblast, ectoplacental cone, and decidua (Constam et al. 1996; Rancourt and Rancourt 1997). We show here that an epiblast-specific conditional deletion of Pcsk5 exon1 (Meox2Cre; Pcsk $5^{\Delta 1 / f l o x}$ ) recapitulates the cardiac, tracheoesophageal, and anorectal malformations, hindlimb hypoplasia, presacral mass, exomphalos, renal and palatal agenesis, and pulmonary hypoplasia phenotype. Axial skeletal malformations and renal agenesis in these conditionally deleted embryos have also been reported independently (Essalmani et al. 2008). These results indicate that Pcsk5 functions within the epiblast are necessary for anteroposterior patterning and organogenesis. Notably, embryos with Pcsk $5^{V c c / V c c}$, Meox2Cre; $P \operatorname{csk} 5^{\Delta 1 / f l o x}, P \operatorname{csk} 5^{V c c / \Delta 1}$, and $P \operatorname{csk} 5^{\Delta 1 / \Delta 1}$ genotypes all survive until $15.5 \mathrm{dpc}$ and beyond (Essalmani et al. 2008; this study). However the Pcsk5 exon 4 deletion $\left(P \operatorname{csk} 5^{\Delta 4 / \Delta 4}\right)$ results in embryonic lethality by $4-7 \mathrm{dpc}$ (Essalmani et al. 2006). The mechanism for this earlier embryonic lethality in $P \operatorname{csk} 5^{\Delta 4 / \Delta 4}$ is unclear at present, but may reflect the differing genetic backgrounds of the mice. Alternatively, it is possible that secreted PCSK5 $\Delta 4$ peptide titrates a PCSK5-interacting protein (Essalmani et al. 2006).

The predicted structure of the protein resulting from the mutated gene indicates that the C470R mutation will likely disrupt a predicted disulfide bond in the $\mathrm{P}$ domain. The P domain in the related molecule FURIN is an eight-stranded $\beta$-sandwich that has interdomain contacts with the catalytic domain, and is conserved in PCSK1-7. The predicted effect of the analogous mutation in the FURIN structure is to displace the loop (471482) away from $P \beta 1 \mathrm{a}$. The $\mathrm{P}$ domain is thought to stabilize the catalytic domain, and the propeptide may bind to a crevice formed by the $\mathrm{P}$ domain and the catalytic site (Henrich et al. 2003). Thus, the loss of catalytic activity observed in our in vitro experiments (on BMP4, LEFTY1, and GDF11) is likely a consequence of abnormal P domain folding. As the $\mathrm{P}$ domain is necessary for the intramolecular cleavage and normal trafficking of proprotein convertases through the secretory pathway (Zhou et al. 1998; Ueda et al. 2003), it is likely that the abnormal trafficking of the PCSK5A-C470R mutant observed in our studies (reduced secretion, and ER retention) is a consequence of the perturbed $\mathrm{P}$ domain structure and reduced catalytic activity. Although these results suggest that loss of PCSK5A catalytic activity is the mechanism for the $V c c$ phenotype, an alternative explanation is that the C470R mutation in vivo may simply result in protein degradation as a consequence of misfolding. A limitation of our studies is that available antibodies do not allow us to detect endogenous PCSK5 protein from mouse embryos, and we are unable at present to distinguish between these two alternative explanations.

Genetic evidence indicates that certain PCSK5 targets such as BMP4 (Cui et al. 1998) and LEFTY1 (Ulloa et al. 2001) are necessary for normal cardiac development (Meno et al. 1998; Jiao et al. 2003; Liu et al. 2004; Goldman et al. 2006). Our studies showed that PCSK5AC470R does not cleave either BMP4 or LEFTY1 in cotransfection assays. BMP4 deficiency is associated with cardiac abnormalities that include common arterial trunk, and atrioventricular septation defects (Jiao et al. 
2003; Liu et al. 2004), both abnormalities being observed in Pcsk5 mutant embryos. Importantly, BMP4 cleavage is essential for ventral body wall closure (mutation resulting in omphalocoele/exomphalos), and cardiac development (Goldman et al. 2006). These features (exomphalos and abnormal cardiac development) were observed in the Pcsk5 mutants. Cardiac malformations in LEFTY1 deficiency include double-outlet right ventricle, transposition of great arteries, atrioventricular septal defect, and right aortic arch (Meno et al. 1998). These malformations were also observed in Pcsk5 mutants. Thus it is possible that cardiac malformations in the $V c c$ mutant arise, at least in part, as a result of abnormal BMP4 or LEFTY1 cleavage.

Certain features of the Pcsk $5^{V c c / V c c}$ mutant-palatal agenesis, renal agenesis, increased numbers of thoracic vertebrae to 18 , increased numbers of true ribs-phenocopy the loss of Gdf11 (McPherron et al. 1999; Esquela and Lee 2003). Our results presented here extend the known phenotypic features of Gdf11 deficiency, and show that there are further points of similarity with Pcsk5 deficiency; i.e., Gdf11 deficiency also results in a presacral mass arising from the spinal cord, exomphalos, and anorectal malformation. We showed that GDF11 peptide can be cleaved by PCSK5A, that this activates GDF11-mediated signaling, and that $G d f 11$ expression is not deficient in the Pcsk5 $5^{V c c / V c c}$ mutant. Moreover, Gdf11 and Pcsk5 are expressed in close physical proximity in the developing embryo. Gdf11 is expressed in the tailbud and neural tube, whereas Pcsk5 is expressed in the adjacent caudal somites (Constam et al. 1996; McPherron et al. 1999; Nakashima et al. 1999; Essalmani et al. 2006; this study). Similarly in limb buds, while Gdf11 is expressed in the mesenchyme, Pcsk5 mRNA localizes to the overlying apical ectodermal ridge. This situation is reminiscent of the activation of the related NODAL precursor in the epiblast by the proprotein convertases FURIN and PACE4, which are provided by cells in the adjacent extraembryonic ectoderm and visceral endoderm (Beck et al. 2002; Mesnard et al. 2006).

GDF11 is a TGF $\beta$ superfamily molecule (McPherron et al. 1999|, and analysis of both human and mouse GDF11 peptides indicates that they have a completely conserved consensus proprotein convertase cleavage site (after residue 298 in the human peptide; i.e., NTKRSRR $\downarrow$ NL) (Duckert et al. 2004). This supports the idea that both mouse and human GDF11 are PCSK5A targets. Independent experiments have shown that selectivity of PCSK5A for GDF11 is mediated by the Asn residue immediately following the cleavage site, and that other proprotein convertases (FURIN, PCSK7 [PC7] or PCSK6 [PACE4]) will not cleave GDF11 (Essalmani et al. 2008). Taken together, these results indicate that Pcsk5 functions at least in part by activating GDF11 signaling in the developing embryo. Consistent with this, mutation in $G d f 11$, in the type II receptor Acvr2b, and the type I receptor $A l k 5$, are associated with a caudal phenotype involving increased numbers of thoracic vertebrae, and renal agenesis, and are associated with abnormal expression of Hoxc6, Hoxc8, Hoxc10, and Hoxc11 (Oh and Li
1997; McPherron et al. 1999; Oh et al. 2002; Esquela and Lee 2003; Andersson et al. 2006).

The regulation of Hox expression is complex, involving GDF11 and BMPs (McPherron et al. 1999; Li and Cao 2006), TGF $\beta$ receptors (Oh and Li 1997; Oh et al. 2002; Andersson et al. 2006), BMP receptors (Ovchinnikov et al. 2006), retinoic acid receptors (Daftary and Taylor 2006), chromatin modifying Polycomb/Trithorax genes (Ringrose and Paro 2007), and noncoding transcripts that arise at the Hox locus (Rinn et al. 2007; Sessa et al. 2007). Genetic evidence shows that Hox10acd is necessary for the suppression of thoracic ribs on lumbar and sacral vertebrae, and formation of the hindlimb stylopod (femur, patella) (Wellik and Capecchi 2003). Hox11acd is necessary for the formation of the sacrum and the hindlimb zeugopod (tibia, fibula) (Wellik and Capecchi 2003), and the metanephric kidney (Wellik et al. 2002). Hoxd12 is necessary for normal anorectal development (Kondo et al. 1996). Our experiments establish a new mechanism for the coordinated regulation of paralogous caudal Hox gene expression. We observed reduction of Hox10acd expression in Pcsk5 mutant embryos. This could explain the increased numbers of thoracic vertebrae, hypoplastic femur, and absent patella. The reduction of Hox11ad in Pcsk5 mutant embryos (most marked in the caudal region and the hindlimb bud), could explain the absence of sacrum formation, abnormal tibia and fibula patterning, and renal agenesis. The reduction of Hoxd12 would explain the anorectal malformations. The relatively normal expression of $T b x 6$ and $T$, genes also important in renal, vertebral, and caudal patterning (Showell et al. 2004), suggest that these are unlikely to contribute to the Pcsk 5 mutant phenotype. In addition, our results show that Pcsk 5 also regulates expression of a noncoding transcript Hoxa11os (Hsieh-Li et al. 1995). While the function of this transcript is not known, other noncoding Hox locus transcripts have been shown to regulate Hox expression (Rinn et al. 2007).

Our results also show that $M n x 1$ (Hlxb9, Hb9), the mouse homolog of $H L X B 9$, is misexpressed in the tail bud region of Pcsk5 mutant embryos. Mnx1 specifies motor neurons and the dorsal pancreas (Arber et al. 1999; Harrison et al. 1999; Li et al. 1999), and ectopic expression triggers motorneuron differentiation (Arber et al. 1999). The ectopic Mnx1 expression observed could explain the presacral neural-derived mass observed in RECC/19 embryos. A characteristic feature of CS is this presacral mass (Currarino et al. 1981; Lynch et al. 2000), and it is notable that HLXB9 mutations are associated with CS/Autosomal-dominant sacral agenesis (Ross et al. 1998; Lynch et al. 2000), suggesting a link.

The features observed in the mouse embryos recapitulate many aspects of the VACTERL association, and also of CRS and CS, syndromes that have significant overlap with VACTERL. Specifically, the mouse mutant has vertebral, anorectal, cardiac, tracheoesophageal, renal, and limb malformations observed in VACTERL association. The mouse mutant also recapitulates anomalies associated with CRS-i.e., sacral agenesis, spinal cord anomalies, and pulmonary hypoplasia. In addition, it recapitu- 
lates the presacral mass, a feature specific to CS. These shared features suggested that Pcsk5 may play a role in these human syndromes. Our preliminary investigations in this regard support this idea as they identify certain heterozygous variants in evolutionarily conserved residues of PCSK5. These variants are present in patients with VACTERL/CRS phenotypes in humans but are absent in ethnically matched controls indicating that they are unlikely to be common polymorphisms. A confounding factor is that the mutations are inherited from apparently normal parents. This raises the possibility that the phenotype results from additional environmental modifiers such as maternal diabetes (Kousseff 1999; Aberg et al. 2001; Loffredo et al. 2001), and genetic modifiers; e.g., within the PCSK5 genetic network. The functional significance of these mutations is unclear at present, and further work is necessary to determine this.

Taken together, these studies extend our understanding of the role of proprotein convertases in the patterning of the mammalian embryo. Other members of the Pcsk family that have been shown to control embryonic patterning are Pcsk6 (SPC4/PACE4), which controls leftright patterning and anterior central nervous system development (Constam and Robertson 2000a), and Furin (Pcsk3/PACE/SPC1), which controls cardiac tube fusion and looping, embryo turning, ventral body wall closure, and yolk sac vasculature development (Roebroek et al. 1998; Constam and Robertson 2000b). In contrast, Pcsk5 has unique roles in controlling anteroposterior axial patterning, coordinated regulation of caudal Hox expression, neural and gut development, and nephrogenesis. Our results support the idea that activation of GDF11 by PCSK5 is likely necessary for normal anteroposterior patterning, nephrogenesis, and limb and anorectal development. They also suggest that activation of BMP4 and LEFTY1 by PCSK5 may play a role in normal cardiac development. The identification of nonsynonymous mutations in PCSK5B in patients with VACTERL and CRS suggests that the PCSK5 genetic network may play a role in the pathogenesis of these syndromes. In summary, our data establishes novel and pleiotropic functions for Pcsk5 in mammalian development and the coordinated expression of caudal Hox genes. We propose that Pcsk5, at least in part via GDF11, coordinately regulates the expression of caudal Hox paralogs, to control anteroposterior patterning, nephrogenesis, and limb and anorectal development.

\section{Materials and methods}

Mice and embryos

Standard methods were used to create ENU mutant mice and genetically map the $V c c$ mutation (see the Supplemental Material). MRI and skeletal preparations were performed as described (Kaufman 1994; Schneider et al. 2004). The generation of Pcsk5 $5^{\text {flox/+ }}$ and Pcsk $5^{\Delta 1}$ alleles is described elsewhere (Essalmani et al. 2008). Gdf11 knockout mice were a kind gift from Se-Jin Lee (Johns Hopkins University, Baltimore, MD).

\section{Molecular biology}

Standard molecular cloning techniques were used (Ausubel et al. 1995).

\section{Plasmids}

We obtained mouse IMAGE clone 4036159 (CMV-PCSK5A) from Geneservice Ltd., and introduced a C-terminal Flag tag and the C470R mutation using PCR. PMT23-BMP2-Myc-tagged BMP4 was a kind gift from Tom Jessel (Columbia University). CMV-SPORT6-human-GDF11 plasmid was subcloned from a GDF11 plasmid (kind gift from Se-Jin Lee). AR3-lux reporter plasmid has been described previously (Yan et al. 2002). LEFTY1 plasmid was a gift from $\mathrm{H}$. Hamada (Osaka University). We obtained Hoxc10, Hoxa11, Mnx1, and Tbx6 mouse plasmids from Geneservices Ltd. (IMAGE: 30059581, 1095072, 40130560, 636895). Hoxa10 is a gift from P. Gruss and A. Mansouri, Hoxd11 is a gift from D. Duboule, and Hoxd10 is a gift from D. Bogani. Hoxa11os plasmid was created by PCR and is common for opposite strand transcripts 3C, 23A, 59, and 40 (Hsieh-Li et al. 1995). $T$ plasmid was a gift from B. Hermann.

\section{Cell-based assays}

COS-1 and 293T cells were transfected with Fugene (Roche), and HEK293 cells were transfected with Effectene (Qiagen). Monoclonal M2 anti-Flag antibody was obtained from Sigma, anti-myc mouse monoclonal 9E10 was obtained from Roche, goat anti-mouse HRP conjugate was obtained from DAKO, LEFTY1 polyclonal rabbit antibody was obtained from Santa Cruz Biotechnologies (sc-7408), sheep anti-human TGN46 polyclonal antibody was obtained from Serotec, rabbit anti-calnexin antibody was obtained from Abcam (ab13504-100), and secondary Alexa Fluor488 goat anti-mouse-conjugated (green) and Alexa Fluor594 donkey anti-sheep-conjugated (red) antibodies were obtained from Invitrogen. Western blot analysis of wholecell lysates and serum-free conditioned medium were performed as described (Beck et al. 2002). Radioimmunoprecipitation assays were performed as described (Essalmani et al. 2006). The luciferase reporter assay was performed as described (Yan et al. 2002; Andersson et al. 2006). We performed immunofluorescence using standard techniques (Ausubel et al. 1995), and data were collected sequentially using a Zeiss confocal microscope. Peptide cleavage studies were performed as described (Essalmani et al. 2006, 2008).

\section{In situ hybridization}

In situ hybridization was performed as described previously (Bamforth et al. 2004).

\section{Human mutations}

Details are provided in the legend for Supplemental Figure 6.

\section{Acknowledgments}

These studies were funded by BHF grant PG/04/023/16761, the MRC, CIHR grant MOP 44363, Canada chair 201652, and Wellcome Trust Senior Research Fellowship 054528. We thank Se-Jin Lee for the gift of Gdf11 knockout mice; S. Wells, M. Cormack, and S. Polley for help with dissections and mouse colony maintenance; H. Barnes for help with MRI; L. Winchester for microarray analysis; D. Brooker for pyrosequencing; L. Buckingham and $\mathrm{H}$. Butler for iPLEX assays; J. Majewska for help with 3D reconstructions; Z. Holloway for advice on antibodies and immunofluorescence; A. Pasquato for in vitro PCSK5 activity tests; D. Donnai, H. Murphy, M. Parker, E. Roper, E. Blair, H. Stewart, and M. Gatzoulis for providing patient samples; and all colleagues who generously sent us plas- 
mids. S.B. is a Wellcome Trust Senior Research Fellow in Clinical Science.

\section{References}

Aberg, A., Westbom, L., and Kallen, B. 2001. Congenital malformations among infants whose mothers had gestational diabetes or preexisting diabetes. Early Hum. Dev. 61: 85-95.

Andersson, O., Reissmann, E., and Ibanez, C.F. 2006. Growth differentiation factor 11 signals through the transforming growth factor- $\beta$ receptor ALK5 to regionalize the anteriorposterior axis. EMBO Rep. 7: 831-837.

Arber, S., Han, B., Mendelsohn, M., Smith, M., Jessell, T.M., and Sockanathan, S. 1999. Requirement for the homeobox gene $\mathrm{Hb} 9$ in the consolidation of motor neuron identity. Neuron 23: 659-674.

Ausubel, F., Brent, R., Kingston, R.E., Moore, D.D., Seidman, J.G., Smith, J.A., and Struhl, K. 1995. Short protocols in molecular biology. John Wiley \& Sons, Inc., New York.

Bamforth, S.D., Braganca, J., Farthing, C.R., Schneider, J.E., Broadbent, C., Michell, A.C., Clarke, K., Neubauer, S., Norris, D., Brown, N.A., et al. 2004. Cited2 controls left-right patterning and heart development through a Nodal-Pitx2c pathway. Nat. Genet. 36: 1189-1196.

Beck, S., Le Good, J.A., Guzman, M., Ben Haim, N., Roy, K., Beermann, F., and Constam, D.B. 2002. Extraembryonic proteases regulate Nodal signalling during gastrulation. Nat. Cell Biol. 4: 981-985.

Bogani, D., Willoughby, C., Davies, J., Kaur, K., Mirza, G., Paudyal, A., Haines, H., McKeone, R., Cadman, M., Pieles, G., et al. 2005. Dissecting the genetic complexity of human $6 \mathrm{p}$ deletion syndromes by using a region-specific, phenotypedriven mouse screen. Proc. Natl. Acad. Sci. 102: 12477-12482.

Constam, D.B. and Robertson, E.J. 2000a. SPC4/PACE4 regulates a TGF $\beta$ signaling network during axis formation. Genes \& Dev. 14: 1146-1155.

Constam, D.B. and Robertson, E.J. 2000b. Tissue-specific requirements for the proprotein convertase furin/SPC1 during embryonic turning and heart looping. Development 127: $245-254$

Constam, D.B., Calfon, M., and Robertson, E.J. 1996. SPC4, SPC6, and the novel protease SPC7 are coexpressed with bone morphogenetic proteins at distinct sites during embryogenesis. J. Cell Biol. 134: 181-191.

Cui, Y., Jean, F., Thomas, G., and Christian, J.L. 1998. BMP-4 is proteolytically activated by furin and/or PC6 during vertebrate embryonic development. EMBO J. 17: 4735-4743.

Currarino, G., Coln, D., and Votteler, T. 1981. Triad of anorectal, sacral, and presacral anomalies. AJR Am. J. Roentgenol. 137: 395-398.

Daftary, G.S. and Taylor, H.S. 2006. Endocrine regulation of HOX genes. Endocr. Rev. 27: 331-355.

De Bie, I., Marcinkiewicz, M., Malide, D., Lazure, C., Nakayama, K., Bendayan, M., and Seidah, N.G. 1996. The isoforms of proprotein convertase PC5 are sorted to different subcellular compartments. J. Cell Biol. 135: 1261-1275.

Duckert, P., Brunak, S., and Blom, N. 2004. Prediction of proprotein convertase cleavage sites. Protein Eng. Des. Sel. 17: 107-112.

Ellies, L.G., Tsuboi, S., Petryniak, B., Lowe, J.B., Fukuda, M., and Marth, J.D. 1998. Core 2 oligosaccharide biosynthesis distinguishes between selectin ligands essential for leukocyte homing and inflammation. Immunity 9: 881-890.

Esquela, A.F. and Lee, S.J. 2003. Regulation of metanephric kid- ney development by growth/differentiation factor 11 . Dev Biol. 257: 356-370.

Essalmani, R., Hamelin, J., Marcinkiewicz, J., Chamberland, A., Mbikay, M., Chretien, M., Seidah, N.G., and Prat, A. 2006. Deletion of the gene encoding proprotein convertase $5 / 6$ causes early embryonic lethality in the mouse. Mol. Cell. Biol. 26: 354-361.

Essalmani, R., Marcinkiewicz, J., Chamberland, A., Pasquato, A., Seidah, N.G., and Prat, A. 2008. In vivo functions of the proprotein convertase PC5/6 during mouse development: Gdf11 is a likely substrate. Proc. Natl. Acad. Sci. doi: 10.1073/pnas.0709428105.

Garcia-Garcia, M.J., Eggenschwiler, J.T., Caspary, T., Alcorn, H.L., Wyler, M.R., Huangfu, D., Rakeman, A.S., Lee, J.D., Feinberg, E.H., Timmer, J.R., et al. 2005. Analysis of mouse embryonic patterning and morphogenesis by forward genetics. Proc. Natl. Acad. Sci. 102: 5913-5919.

Goldman, D.C., Hackenmiller, R., Nakayama, T., Sopory, S., Wong, C., Kulessa, H., and Christian, J.L. 2006. Mutation of an upstream cleavage site in the BMP4 prodomain leads to tissue-specific loss of activity. Development 133: 1933-1942.

Harrison, K.A., Thaler, J., Pfaff, S.L., Gu, H., and Kehrl, J.H. 1999. Pancreas dorsal lobe agenesis and abnormal islets of Langerhans in Hlxb9-deficient mice. Nat. Genet. 23: 71-75.

Henrich, S., Cameron, A., Bourenkov, G.P., Kiefersauer, R., Huber, R., Lindberg, I., Bode, W., and Than, M.E. 2003. The crystal structure of the proprotein processing proteinase furin explains its stringent specificity. Nat. Struct. Biol. 10: 520-526.

Henrich, S., Lindberg, I., Bode, W., and Than, M.E. 2005. Proprotein convertase models based on the crystal structures of furin and kexin: Explanation of their specificity. J. Mol. Biol. 345: 211-227.

Herrmann, M.G., Durtschi, J.D., Bromley, L.K., Wittwer, C.T., and Voelkerding, K.V. 2006. Amplicon DNA melting analysis for mutation scanning and genotyping: Cross-platform comparison of instruments and dyes. Clin. Chem. 52: 494 503.

Herron, B.J., Lu, W., Rao, C., Liu, S., Peters, H., Bronson, R.T., Justice, M.J., McDonald, J.D., and Beier, D.R. 2002. Efficient generation and mapping of recessive developmental mutations using ENU mutagenesis. Nat. Genet. 30: 185-189.

Hsieh-Li, H.M., Witte, D.P., Weinstein, M., Branford, W., Li, H., Small, K., and Potter, S.S. 1995. Hoxa 11 structure, extensive antisense transcription, and function in male and female fertility. Development 121: 1373-1385.

Jiao, K., Kulessa, H., Tompkins, K., Zhou, Y., Batts, L., Baldwin, H.S., and Hogan, B.L. 2003. An essential role of Bmp4 in the atrioventricular septation of the mouse heart. Genes \& Dev. 17: 2362-2367.

Kaufman, M.H. 1994. The atlas of mouse development. Academic Press, London.

Khoury, M.J., Cordero, J.F., Greenberg, F., James, L.M., and Erickson, J.D. 1983. A population study of the VACTERL association: Evidence for its etiologic heterogeneity. Pediatrics 71: 815-820.

Kondo, T., Dolle, P., Zakany, J., and Duboule, D. 1996. Function of posterior HoxD genes in the morphogenesis of the anal sphincter. Development 122: 2651-2659.

Kousseff, B.G. 1999. Gestational diabetes mellitus (class A): A human teratogen? Am. I. Med. Genet. 83: 402-408.

Li, X. and Cao, X. 2006. BMP signaling and skeletogenesis. Ann. N. Y. Acad. Sci. 1068: 26-40.

Li, H., Arber, S., Jessell, T.M., and Edlund, H. 1999. Selective agenesis of the dorsal pancreas in mice lacking homeobox 
gene Hlxb9. Nat. Genet. 23: 67-70.

Liu, W., Selever, J., Wang, D., Lu, M.F., Moses, K.A., Schwartz, R.J., and Martin, J.F. 2004. Bmp4 signaling is required for outflow-tract septation and branchial-arch artery remodeling. Proc. Natl. Acad. Sci. 101: 4489-4494.

Loffredo, C.A., Wilson, P.D., and Ferencz, C. 2001. Maternal diabetes: An independent risk factor for major cardiovascular malformations with increased mortality of affected infants. Teratology 64: 98-106.

Lynch, S.A., Wang, Y., Strachan, T., Burn, J., and Lindsay, S. 2000. Autosomal dominant sacral agenesis: Currarino syndrome. J. Med. Genet. 37: 561-566.

McPherron, A.C., Lawler, A.M., and Lee, S.J. 1999. Regulation of anterior/posterior patterning of the axial skeleton by growth/differentiation factor 11. Nat. Genet. 22: 260-264.

Meno, C., Shimono, A., Saijoh, Y., Yashiro, K., Mochida, K., Ohishi, S., Noji, S., Kondoh, H., and Hamada, H. 1998. lefty- 1 is required for left-right determination as a regulator of lefty-2 and nodal. Cell 94: 287-297.

Mesnard, D., Guzman-Ayala, M., and Constam, D.B. 2006. Nodal specifies embryonic visceral endoderm and sustains pluripotent cells in the epiblast before overt axial patterning. Development 133: 2497-2505

Morris, A.P. 2006. A flexible Bayesian framework for modeling haplotype association with disease, allowing for dominance effects of the underlying causative variants. Am. J. Hum. Genet. 79: 679-694.

Nakashima, M., Toyono, T., Akamine, A., and Joyner, A.L. 1999. Expression of growth/differentiation factor 11, a new member of the BMP/TGF $\beta$ superfamily during mouse embryogenesis. Mech. Dev. 80: 185-189.

Oh, S.P. and Li, E. 1997. The signaling pathway mediated by the type IIB activin receptor controls axial patterning and lateral asymmetry in the mouse. Genes \& Dev. 11: 1812-1826.

Oh, S.P., Yeo, C.Y., Lee, Y., Schrewe, H., Whitman, M., and Li, E. 2002. Activin type IIA and IIB receptors mediate Gdf11 signaling in axial vertebral patterning. Genes \& Dev. 16: $2749-2754$.

Ovchinnikov, D.A., Selever, J., Wang, Y., Chen, Y.T., Mishina, Y., Martin, J.F., and Behringer, R.R. 2006. BMP receptor type IA in limb bud mesenchyme regulates distal outgrowth and patterning. Dev. Biol. 295: 103-115.

Papathanasiou, P. and Goodnow, C.C. 2005. Connecting mammalian genome with phenome by ENU mouse mutagenesis: Gene combinations specifying the immune system. Annu. Rev. Genet. 39: 241-262.

Petrini, J., Damus, K., Russell, R., Poschman, K., Davidoff, M.J., and Mattison, D. 2002. Contribution of birth defects to infant mortality in the United States. Teratology 66 (Suppl. 1): S3-S6. doi: 10.1002/tera.90002.

Rancourt, S.L. and Rancourt, D.E. 1997. Murine subtilisin-like proteinase SPC6 is expressed during embryonic implantation, somitogenesis, and skeletal formation. Dev. Genet. 21: $75-81$.

Ringrose, L. and Paro, R. 2007. Polycomb/Trithorax response elements and epigenetic memory of cell identity. Development 134: 223-232.

Rinn, J.L., Kertesz, M., Wang, J.K., Squazzo, S.L., Xu, X., Brugmann, S.A., Goodnough, L.H., Helms, J.A., Farnham, P.J., Segal, E., et al. 2007. Functional demarcation of active and silent chromatin domains in human HOX loci by noncoding RNAs. Cell 129: 1311-1323.

Roebroek, A.J., Umans, L., Pauli, I.G., Robertson, E.J., van Leuven, F., Van de Ven, W.J., and Constam, D.B. 1998. Failure of ventral closure and axial rotation in embryos lacking the proprotein convertase Furin. Development 125: 4863-4876.
Ross, A.J., Ruiz-Perez, V., Wang, Y., Hagan, D.M., Scherer, S., Lynch, S.A., Lindsay, S., Custard, E., Belloni, E., Wilson, D.I., et al. 1998. A homeobox gene, HLXB9, is the major locus for dominantly inherited sacral agenesis. Nat. Genet. 20: 358361.

Schneider, J.E., Bose, J., Bamforth, S.D., Gruber, A.D., Broadbent, C., Clarke, K., Neubauer, S., Lengeling, A., and Bhattacharya, S. 2004. Identification of cardiac malformations in mice lacking Ptdsr using a novel high-throughput magnetic resonance imaging technique. BMC Dev. Biol. 4: 16. doi: 10.1186/1471-213X-4-16.

Seidah, N.G. and Chretien, M. 1999. Proprotein and prohormone convertases: A family of subtilases generating diverse bioactive polypeptides. Brain Res. 848: 45-62.

Sessa, L., Breiling, A., Lavorgna, G., Silvestri, L., Casari, G., and Orlando, V. 2007. Noncoding RNA synthesis and loss of Polycomb group repression accompanies the colinear activation of the human HOXA cluster. RNA 13: 223-239.

Showell, C., Binder, O., and Conlon, F.L. 2004. T-box genes in early embryogenesis. Dev. Dyn. 229: 201-218.

Ueda, K., Lipkind, G.M., Zhou, A., Zhu, X., Kuznetsov, A., Philipson, L., Gardner, P., Zhang, C., and Steiner, D.F. 2003. Mutational analysis of predicted interactions between the catalytic and P domains of prohormone convertase 3 (PC3/ PC1). Proc. Natl. Acad. Sci. 100: 5622-5627.

Ulloa, L., Creemers, J.W., Roy, S., Liu, S., Mason, J., and Tabibzadeh, S. 2001. Lefty proteins exhibit unique processing and activate the MAPK pathway. J. Biol. Chem. 276: 21387-21396.

Walder, R.Y., Landau, D., Meyer, P., Shalev, H., Tsolia, M., Borochowitz, Z., Boettger, M.B., Beck, G.E., Englehardt, R.K., Carmi, R., et al. 2002. Mutation of TRPM6 causes familial hypomagnesemia with secondary hypocalcemia. Nat. Genet. 31: 171-174.

Wellik, D.M. and Capecchi, M.R. 2003. Hox10 and Hox11 genes are required to globally pattern the mammalian skeleton. Science 301: 363-367.

Wellik, D.M., Hawkes, P.J., and Capecchi, M.R. 2002. Hox11 paralogous genes are essential for metanephric kidney induction. Genes \& Dev. 16: 1423-1432.

Xiang, Y., Molloy, S.S., Thomas, L., and Thomas, G. 2000. The PC6B cytoplasmic domain contains two acidic clusters that direct sorting to distinct trans-Golgi network/endosomal compartments. Mol. Biol. Cell 11: 1257-1273.

Yan, Y.T., Liu, J.J., Luo, Y.E.C., Haltiwanger, R.S., Abate-Shen, C., and Shen, M.M. 2002. Dual roles of Cripto as a ligand and coreceptor in the nodal signaling pathway. Mol. Cell. Biol. 22: 4439-4449.

Yu, Q., Shen, Y., Chatteriee, B., Siegfried, B.H., Leatherbury, L., Rosenthal, J., Lucas, J.F., Wessels, A., Spurney, C.F., Wu, Y.J., et al. 2004. ENU induced mutations causing congenital cardiovascular anomalies. Development 131: 6211-6223.

Zhou, A., Martin, S., Lipkind, G., LaMendola, J., and Steiner, D.F. 1998. Regulatory roles of the P domain of the subtilisinlike prohormone convertases. J. Biol. Chem. 273: 11107-11114. 


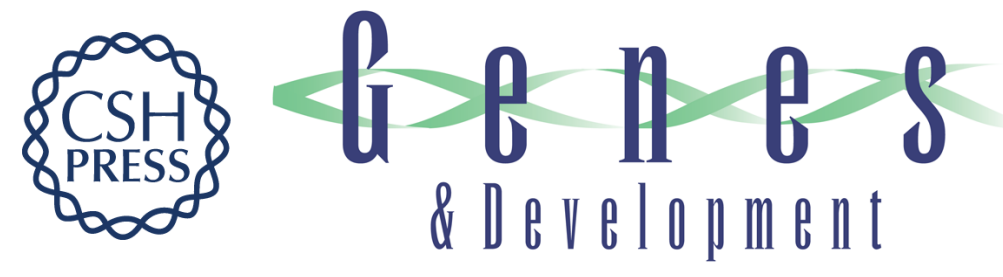

\section{VACTERL/caudal regression/Currarino syndrome-like malformations in mice with mutation in the proprotein convertase Pcsk5}

Dorota Szumska, Guido Pieles, Rachid Essalmani, et al.

Genes Dev. 2008, 22:

Access the most recent version at doi:10.1101/gad.479408

Supplemental
Material http://genesdev.cshlp.org/content/suppl/2008/05/21/22.11.1465.DC1

References This article cites 63 articles, 30 of which can be accessed free at:

http://genesdev.cshlp.org/content/22/11/1465.full.html\#ref-list-1

License Freely available online through the Genes \& Development Open Access option.

Email Alerting Receive free email alerts when new articles cite this article - sign up in the box at the top

Service right corner of the article or click here.

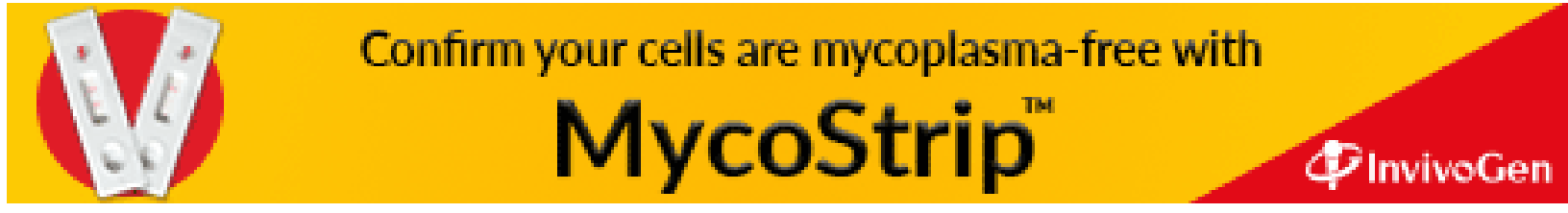

